\title{
Association Between Maximum Standardize Uptake Value and Prognostic Factors at Endometrioid Type Endometrial Carcinoma
}

\author{
Varol GULSEREN', Mustafa KOCAER ${ }^{2}$, Isa Aykut OZDEMIR ${ }^{3}$, Ilker CAKIR², Mehmet GOKCU², \\ Ozgu GUNGORDUK ${ }^{4}$, Muzaffer SANCI ${ }^{2}$, Kemal GUNGORDUK ${ }^{4}$
}

Mersin, Turkey

\begin{abstract}
OBJECTIVE: Investigate the relationship between the maximum standardize uptake value (SUVmax) values and the prognostic factors in endometrioid-type endometrial cancer (EEC) patients undergoing preoperative positron emission tomography / computed tomography (PET/CT).

STUDY DESIGN: We reviewed retrospectively the records of patients with EEC diagnosis who underwent hysterectomy in Gynecologic Oncology Clinic of Tepecik Training and Research Hospital between January 2010 and January 2017 in this retrospective study. Receiver operating characteristic (ROC) curve analysis was performed to determine the cut-off values of SUVmax for predicting clinical parameters. The area under the ROC curve (AUC) is presented as a measure of discrimination.
\end{abstract}

RESULTS: It was calculated that the SUVmax values in the uterine tumor were higher and statistically significant in the presence of advanced stage (III-IV), histologic grade III, deep myometrial invasion ( $\geq 1$ / 2) and large tumor size $(\geq 4 \mathrm{~cm})$ from prognostic factors. The SUVmax values of the groups with and without cervical invasion did not different from each other. In order to use the SUVmax value as a diagnostic test in the ROC analysis, the AUC values were as follows; the grade of advanced stage tumor was 0,685 , the grade 3 tumor was 0,797 , the depth of myometrial invasion was 0,781 , and the size of the large tumor was 0,905.

CONCLUSION: SUVmax value in primary uterine tumor was found to be higher in prognostic factors in patients with advanced stage, high grade, deep myometrial invasion and large tumor.

Keywords: Endometrial carcinoma, Standardize uptake value, Positron emission tomography / computed tomography

Gynecol Obstet Reprod Med 2020;26(1):58-63

${ }^{1}$ Mersin State Hospital Department of Obstetrics and Gynecology, Mersin,

${ }^{2}$ University of Health Sciences Tepecik Education and Research Hospital Department of Gynecologic Oncology, Izmir

3 University of Health Sciences, Bakirkoy Sadi Konuk Research and Training Hospital Department of Gynecology and Oncology, Istanbul

${ }^{4}$ Mugla Sitkı Koçman University Education and Research Hospital, Department of Gynecology and Oncology, Mugla

Address of Correspondence: Varol Gulseren

Mersin State Hospital Department of

Obstetrics and Gynecology 96015 Street 33240 Mersin, Turkey

varolgulseren@dr.com

Submitted for Publication: Revised for Publication:

15.07.2018

20.12 .2018

Accepted for Publication:

07.01 .2019

ORCID IDs of the authors: MK:0000-0003-1813-0500, IC:0000-0003-0815-0954, OG:0000-0003-2665-0936 KG:0000-0002-2325-1756

\begin{tabular}{|c|c|}
\hline Quick Response Code: & Access this article online \\
\cline { 2 - 2 } & Website: www.gorm.com.tr \\
\hline & e- mail: info@gorm.com.tr \\
\cline { 2 - 3 } & DOI:10.21613/GORM.2018.817 \\
\hline
\end{tabular}

How to cite this article: Gulseren V. Kocaer M. Ozdemir IA, Cakir I. Gokcu M. Gungorduk O. Sanci M. Gungorduk K. Association Between Maximum Standardize Uptake Value and Prognostic Factors at Endometrioid Type Endometrial Carcinoma. Gynecol Obstet Reprod Med 2020;26(1):58-63

\section{Introduction}

Endometrial cancer is the most common gynecologic malignancy in developed countries (1-3). The prognosis of patients with endometrioid-type endometrial cancer (EEC) is excellent because they are usually at an early stage when diagnosed (2). When all stages are considered, the 5-year survival rate is $80 \%$ and more (2). Prognosis in EEC is related to stage, age of the patient, histological type and grade of the tumor, cervical invasion, depth of myometrial invasion, lymph node involvement and distant organ metastasis $(1,2)$. There is a significant prognostic difference between the histological types of endometrium carcinoma. EEC type-1 is known as estrogen sensitive endometrial carcinomas and is a good prognostic indicator (3). Surgical staging, as defined by the federation of international gynecology and obstetrics (FIGO), is composed of hysterectomy, bilateral salpingo-oophorectomy, pelvic and paraaortic lymph node dissection, removal of peritoneal cytology, careful exploration of the abdomen, and removal of biopsies from suspected peritoneal surfaces. Stage is considered the most important prognostic factor (3). The most commonly used methods to estimate the preoperative stage of surgery are the 
histological type and grading of the tumor obtained after the histopathological examination of the curettage material and magnetic resonance imaging, which provides better information about solid organs among imaging methods.

Fluorodeoxyglucose positron emission tomography / computed tomography (FDG PET/CT) is a method of imaging using metabolic changes in cancer cells $(2,3)$. Tumor perfusion and metabolism scanning can be performed using carbon11 , fluor-18 (18F), nitrogen-13, oxygen-15 and rubidium-82 radioisotopes (4). $18 \mathrm{~F}-\mathrm{FDG}$ passes through the cell membrane in the same way as glucose but cannot be metabolized by phosphofructokinase-1. Thus, 18F-FDG remaining in the cell reflects glucose uptake into the cell (4). Standardize uptake value (SUV) is a marker of tumor aggression and a marker of metabolic change in cancer tissues (2-4).

The aim of this study was to investigate the relationship between the SUV max values and the prognostic factors (histological grade, depth of myometrial invasion, tumor size, lymph node involvement, cervical invasion, lymphovascular invasion and stage) in EEC patients undergoing preoperative $\mathrm{PET} / \mathrm{CT}$.

\section{Material and Method}

We reviewed retrospectively the records of patients with EEC diagnosis who underwent hysterectomy in Gynecologic Oncology Clinic of Tepecik Training and Research Hospital between January 2010 and January 2017 in this retrospective study. Only patients who underwent preoperative 18F-FDG PET/CT imaging were evaluated and included in the study. Exclusion criteria were serous, clear cell or anaplastic histologic type endometrial carcinomas and patients who did not undergo PET/CT prior to surgery. Tumor staging was performed based on the International Federation of Gynecology and Obstetrics (FIGO) 2009 staging criteria (5). The study was approved by the local ethics committee (Ethics Committee of Katip Çelebi University, Decision number: 45, Date: February 2014). The study was conducted in accordance with the principles of the Declaration of Helsinki.

The diagnosis was established by histopathology in all patients. All surgical specimens were evaluated by specialized gynecologic pathologists. Inclusion criteria were as follows: all types of histology, no intraoperative evidence of extra uterine spread, performance of pelvic and para-aortic lymphadenectomy, and histopathologically proven cervical stromal involvement. Tumor size, grade, histological type, cervical invasion, lymphovascular space invasion (LVSI), lymph node (LN) status, deep of myometrial invasion were analyzed in accordance with the pathology reports. Uterine sections were selected from anterior and posterior aspects of the cervix, lower uterine segment, and uterine corpus. A minimum of 6 sections including the section of deepest tumoral invasion was obtained for all specimens. LVSI was defined as the pres- ence of tumor cells inside the capillary lumens of either the lymphatic or microvascular drainage systems within the primary tumor.

The whole-body 18F-FDG PET/CT images were done using PET/CT scanner (Philips Gemini TF; Philips Healthcare, Andover, MA, USA) which consists of dedicated lutetium orthosilicate full-ring PET scanner and 16-slice CT. Both PET and low-dose CT scanning covered the skull to the proximal thigh. The protocol included $6 \mathrm{~h}$ of fasting before the image acquisition, and all patients were asked to void before undergoing scanning. On the day of the examination, the serum glucose levels measured before 18F-FDG injections were found to be less than $140 \mathrm{mg} / \mathrm{dL}$. Subsequently, $18 \mathrm{~F}$ FDG $(6,5-13,4 \mu \mathrm{Ci})$ was given intravenously 60 to $120 \mathrm{~min}$ before the CT scan, and the patients were instructed to rest in a semi-dark, temperate room between the injection and scanning. At $60 \mathrm{~min}$ after the administration of 18F-FDG, lowdose CT $(50 \mathrm{mAs}, 120 \mathrm{kV})$ covering the area from scull to the proximal thighs was performed to attenuate the correction and precise anatomical localization. Then, an emission scan was conducted in the three-dimensional mode. All images were reconstructed and stored in the axial, coronal, and sagittal slices. The total scanning time was about 20 min per patient. The SUVmax was estimated for each hyper metabolic lesion.

Age, hypertension, presence of diabetes mellitus, gravida, parity information were recorded retrospectively from the files of the patients at the time of diagnosis. Hemogram, hematocrit, platelet, white blood cell, neutrophil, lymphocyte, albumin, gamaglutamyl transferase and CA $125(\mathrm{U} / \mathrm{mL})$ values were examined within the last two weeks before surgery. Complete blood counts were performed with a Coulter LH 750 instrument (Beckman Coulter, Brea; USA) within 1 week prior to operation. If more than one complete blood count result was present, the result closest to the operation date was used for statistical analysis CA-125 values were measured by Roche E170 Moduler System using chemiluminescence method and concentrations were given in $\mathrm{U} / \mathrm{mL}$. Patients who died or left follow-ups on their own desires were excluded from follow-ups.

All surgical operations were carried out by experienced surgeons in the fields of gynecological oncologic surgery. A vertical midline incision was preferred in all patients for ease of access during exploration of the abdomen and organ resection. After entering the peritoneal cavity, peritoneal washing cytology was taken. During exploration of the abdominal cavity, peritoneal surfaces, omentum, colon and small intestine, paracolic, pelvic, mesenteric and para-aortic sites were all examined systematically and palpated to find any suspicious lesions. Hysterectomy was performed in all patients. Pelvic lymphadenectomy consisted of removal of the lymphatic tissue over the external and common iliac vessels and in the obturator fossa. Paraaortic LN dissection was performed by re- 
moving the lymphatic tissue over the inferior vena cava and aorta, beginning at the bifurcation and proceeding to the inferior mesenteric artery if necessary.

\section{Statistical analysis}

Descriptive data were expressed in mean \pm standard deviation and percent. The Student's t-test was used to compare the mean values between two independent groups, while the chi-square $\left(\chi^{2}\right)$ test was used to compare the nominal values between the two groups. Receiver operating characteristic (ROC) curve analysis was performed to determine the cut-off values of SUVmax for predicting clinical parameters. The area under the ROC curve (AUC) is presented as a measure of discrimination. In determining the optimal cut-off values, the Youden index was used. Statistical analysis was performed using the Med-Calc for Windows version 16.0 statistical software (MedCalc Software, Mariakerke, Belgium). A p value of $<0,05$ was considered statistically significant.

\section{Results}

In our study of 149 patients with endometrioid type endometrium cancer who underwent preoperative PET/CT, the clinical characteristics and demographic data of the patients were given in table I. Hysterectomy, bilateral salpingooophorectomy, pelvic and paraaortic lymphadenectomy and peritoneal cytology were performed to all of the patients. Hypertension was found in $44.3 \%$ of the patients and diabetes mellitus was found in $34.0 \%$ of the patients. The mean number of pregnancies was 3.0 2.2 and mean number of deliveries (over 20 weeks) were $2.6 \pm 1.9$. The mean body mass indexes $\left(\mathrm{kg} / \mathrm{m}^{2}\right)$ was $32.5 \pm 5.8$. Myometrial invasion depth was reported to be $\geq 1 / 2$ in 60 patients $(40.3 \%)$. Lymph node metastases were found in 17 patients (11.4\%). Lymphovascular space invasion was positive in 47 (31.5) patients.

The relationship between SUVmax value in primary uterine tumor and the prognostic factors of the patients such as FIGO stage (I-II and III-IV), histological grade (I-II and III), myometrial invasion depth $\geq 1 / 2$, tumor size $\geq 4 \mathrm{~cm}$, cervical stromal invasion status were given in table II. It was calculated that the SUVmax values in the uterine tumor were higher and statistically significant in the presence of advanced stage (III-IV), histologic grade III, deep myometrial invasion $(\geq 1 / 2)$ and large tumor size $(\geq 4 \mathrm{~cm}$ ) from prognostic factors. The SUVmax values of the groups with and without cervical invasion did not different from each other. The usability of SUVmax value as diagnostic test in primary tumor was evaluated by ROC analysis to assess the presence of advanced stage (III-IV), histologic grade III, deep myometrial invasion $(\geq 1 / 2)$ and large tumor size ( $\geq 4 \mathrm{~cm}$ ) (Figure 1). In order to use the SUVmax value as a diagnostic test in the ROC analysis, the area under the curve values were as follows; the grade of advanced stage tumor was 0.685 , the grade 3 tumor was 0.797 , the depth of myometrial invasion was 0.781 , and the size of the large tumor was 0.905 .

Table I: Clinical characteristics of patients in the study group

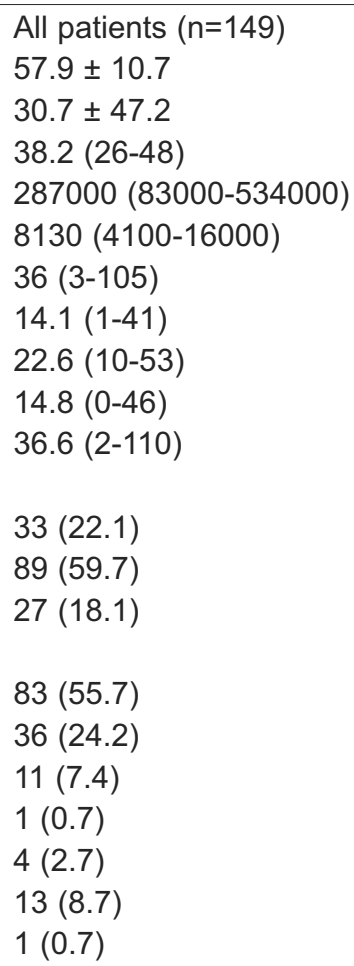


Table II: Relationship between SUVmax values of patients and prognostic factors

\begin{tabular}{lcc}
\hline & $\mathrm{n}(\%)$ & $\begin{array}{c}\text { SUVmax } \\
\text { mean } \pm \text { standard deviation }\end{array}$ \\
\hline FIGO stage & & $13.4 \pm 6.5$ \\
- I-II & $130(87.3)$ & $19.1 \pm 9.1$ \\
- III-IV & $19(12.7)$ & \\
Grade & $122(81.9)$ & $12.7 \pm 6.2$ \\
- I-II & $27(18.1)$ & $20.8 \pm 7.4$ \\
- III & & $11.5 \pm 6.6$ \\
Deep of myometrial invasion & $89(59.7)$ & $18.0 \pm 6.0$ \\
- <1/2 & $60(40.3)$ & \\
- $\geq 1 / 2$ & & $9.5 \pm 4.9$ \\
Size of Tumor & $74(50.4)$ & $18.8 \pm 5.8$ \\
- <4 cm & $75(49.6)$ & $<0.001$ \\
- $\geq 4 \mathrm{~cm}$ & & $14.1 \pm 7.1$ \\
Cervical invasion & $133(89,3)$ & $14.8 \pm 7.4$ \\
-None & $16(10,7)$ & $<.001$ \\
-Yes & & \\
\hline
\end{tabular}

SUVmax: Maximum standardize uptake value, FIGO: Federation of international gynecology and obstetrics

Figure 1: SUVmax value as diagnostic test in primary tumor was evaluated by ROC analysis to assess the prognostic factors

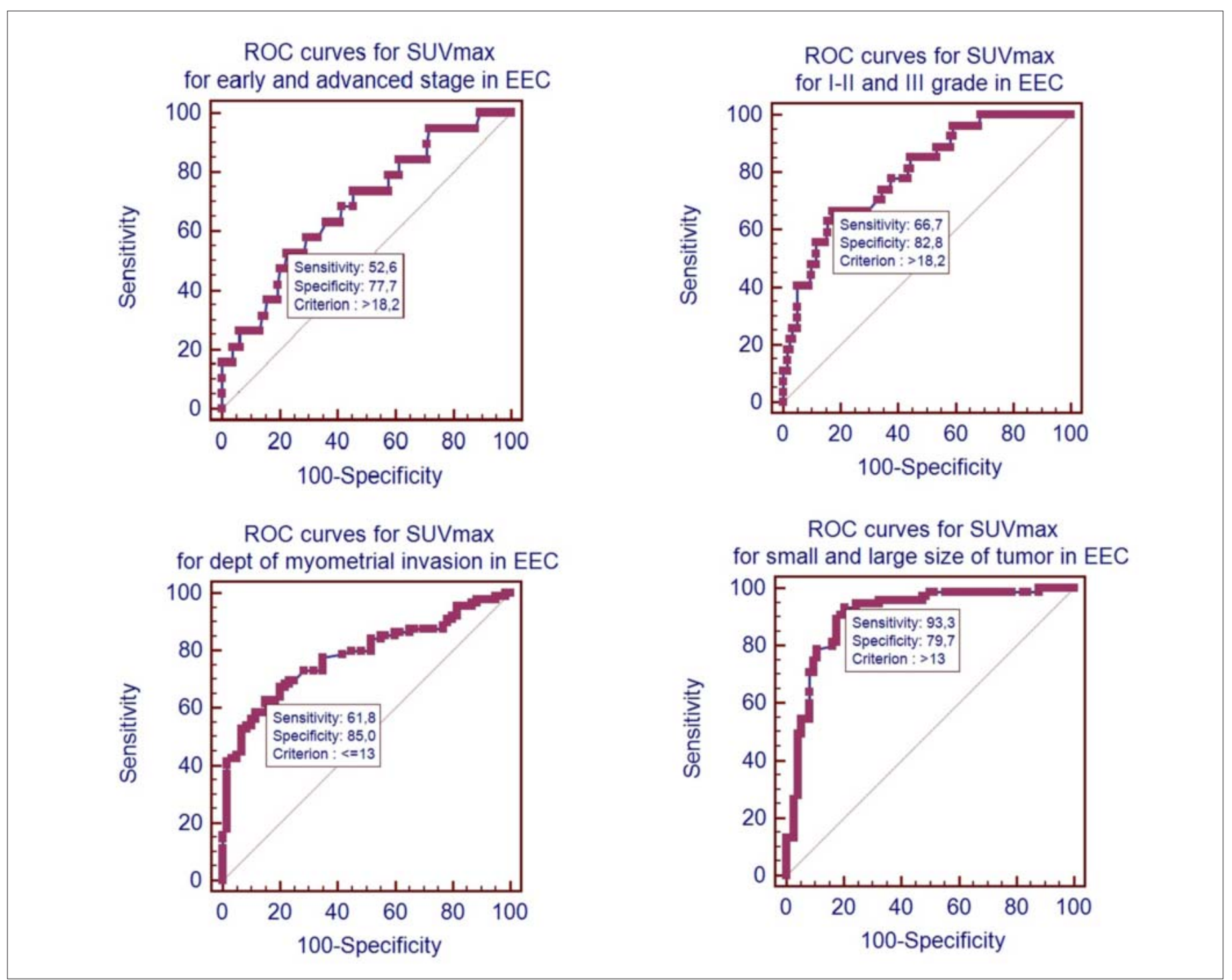




\section{Discussion}

The mean values of SUVmax were compared according to prognostic factors in patients with endometrioid type endometrial cancer who were evaluated by preoperative PET/CT imaging method retrospectively. SUVmax values are generally higher than 2-3 in primary lesions in malignant tumors (3). The high SUVmax value suggests that it is a more aggressive tumor in the PET/CT imaging method based on the mechanism of more glucose use of the tumor cells. As a result of our study, SUVmax value was found to be higher in patients with advanced stage (stage III-IV), grade III tumor, presence of deep myometrial invasion $(\geq 1 / 2)$ and large tumor $(\geq 4 \mathrm{~cm})$.

There are studies in the literature investigating the relationship between the SUVmax values observed in primary tumors and the status of prognostic factors in EEC patients. There are controversies about whether the SUVmax value in the primary tumor differs in some prognostic factors. The mean SUVmax value in the primary uterine tumor grade III $(2,3,6,7)$, advanced stage (III-IV) $(2,6)$, deep myometrial invasion $(6,7,8)$, cervical involvement $(7)$ in patients with nodal metastasis $(2,7)$ and large tumor size $(\geq 4 \mathrm{~cm})(3,8,9)$ was found to be significantly higher in patients with grade III $(2,3,6,7)$, advanced stage (III-IV) $(2,6)$, deep myometrial invasion $(6,7,8)$, cervical involvement $(7)$ in patients with nodal metastasis $(2,7)$ and large tumor size $(\geq 4 \mathrm{~cm})(3,8,9)$. There are also studies that show that SUVmax values are not different in their presence and absence of some of the prognostic factors. The average SUVmax value was not statistically significantly higher in patients with advanced age $(\geq 60)(3,7)$, high grade (III) $(5,8)$, non-endometrioid histological type $(2,7,8)$, postmenopausal status (9), advanced stage (III-IV) (3), deep myometrial invasion (3,9), cervical invasion (3), lymph node metastasis $(3,8,9)$ and lymphovascular space invasion (3). There are studies that found higher or normal-low SUVmax value in primary tumor in endometrial cancer in the presence of high grade (III), advanced stage (III-IV), deep myometrial invasion $(\geq 1 / 2)$, cervical invasion and lymph node involvement. However, studies have shown that SUVmax is higher in the presence of large tumors $(\geq 4 \mathrm{~cm})$, and there is no idea in the opposite direction. In our study, only endometrioid type endometrial cancer patients were examined and the SUVmax value in the primary uterine tumor was calculated to be significantly higher in preoperative PET/CT scans of the patients with advanced stage (III-IV), high grade (III), deep myometrial invasion $(\geq 1 / 2)$. There was no difference in SUVmax values between patients with and without cervical involvement. Although there are studies showing opposite results, it is not a surprising finding that SUVmax value is higher in advanced stage (III-IV), high grade (III), deep myometrial invasion $(\geq 1 / 2)$ and conceived results were found.

Lymph node is not included in the study because it can be examined anatomically in PET / CT. We think that having not a significantly higher SUVmax value in the presence of cervical invasion (it can also be anatomically examined in PET/ CT) is not an important clinical outcome due to similar reasons. The threshold SUVmax value, which could predict the presence of advanced stage (III-IV) and deep myometrial invasion $(\geq 1 / 2)$, was investigated according to ROC curve analysis. It has been found that advanced stage patients can be detected with $72.7 \%$ sensitivity and $68.7 \%$ specificity when the SUVmax value of the primary uterine tumor is 10,4 (area under the curve $=0,721$ ) (2). A smaller area under curve for deep myometrial invasion was found (7). In our study, the threshold values that can be recognized by ROC curve analysis of prognostic factors that differ significantly from each other were investigated. The ideal threshold for SUVmax was 18.2 for recognizing advanced and high grade patients, and the ideal threshold for SUVmax was 13 for detecting deep myometrial invasion and large tumor size. It was not considered contrariwise in other studies that large tumor size with significant difference in SUVmax value had the highest area under curve value (0.905). The SUVmax value $>13$ with a sensitivity of $93.3 \%$ and a specificity of $79.7 \%$ could be a perfect test for large tumor screening in patients.

Nonetheless, there are some missing parts and limitations to this study. One of the most important is the retrospective study design. Due to the retrospective nature of work, incomplete information and records cannot be found in the files. In addition, the low number of patients reduces the power of the study. In addition, a small number of patients over 7 years, being operated by different surgeons and using different treatment regimens, are other limiting factors that can potentially affect the comparison. The main strengths of this study are having reliable data and evaluation of all surgical specimens by experienced gynecologic pathologists and evaluation of PET/CT results by experienced radiologists in the field.

In conclusion, SUVmax value in primary uterine tumor was found to be higher in prognostic factors in patients with advanced stage, high grade, deep myometrial invasion and large tumor. There was no correlation between SUVmax and cervical involvement. The ideal threshold for SUVmax was 18.2 for recognizing advanced and high grade patients, and the ideal threshold for SUVmax was 13 for detecting deep myometrial invasion and large tumor size.

Acknowledgments: None

Financial support or conflict of interest: There is no person/organization that financially supports the study and the authors report no conflicts of interest.

Authors Contributions: VG: Writing. MK: Data collection. IAO: Statistic. IC: Data collection. MG: Control and Editing. OG: Statistic and data collection. MS: Control. KG: Editing.

\section{References}

1. Amant F, Moerman P, Neven P, Timmerman D, Van 
Limbergen E, Vergote I. Endometrial cancer. Lancet. 2005;366(9484):491-505.

2. Ozgü E, Oz M, Yıldız Y, Ozgü BS, Erkaya S and Güngör T. Prognostic value of 18F-FDG PET/CT for identifying high- and low-risk endometrial cancer patients. Ginekol Pol. 2016;87(7):493-7.

3. Nakamura K, Kodama J, Okumura Y, Hongo A, Kanazawa S and Hiramatsu Y. The SUVmax of 18F-FDG PET Correlates with Histological Grade in Endometrial Cancer. Int J Gynecol Cancer. 2010;20(1):110-5.

4. Croteau E, Renaud JM, Richard MA, Ruddy TD, Bénard $\mathrm{F}$ and deKemp RA. PET Metabolic Biomarkers for Cancer. Biomark Cancer. 2016;8(Suppl 2):61-9.

5. Haldorsen IS and Salvesen HB. What Is the Best Preoperative Imaging for Endometrial Cancer? Curr Oncol Rep. 2016;18(4):25.

6. Walentowicz-Sadłecka M, Sadłecki P, Małecki B, Walentowicz P, Marszałek A, Domaracki P, Grabiec M.
SUVmax measured by $18 \mathrm{~F} \mathrm{FDG} \mathrm{PET/CT} \mathrm{in} \mathrm{the} \mathrm{primary}$ tumor in relation to clinical and pathological features of endometrial cancer. Ginekol Pol. 2013;84(9):748-53.

7. Husby J, Reitan B, Biermann M, Trovik J, Bjørge L, Magnussen I, et al. Metabolic Tumor Volume on 18F-FDG PET/CT Improves Preoperative Identification of HighRisk Endometrial Carcinoma Patients. J Nucl Med. 2015; 56(8):1191-8.

8. Shim S, Kim D, Lee D, Lee S, Park J, Lee J, et al. Metabolic tumour volume and total lesion glycolysis, measured using preoperative $18 \mathrm{~F}-\mathrm{FDG} \mathrm{PET} / \mathrm{CT}$, predict the recurrence of endometrial cancer. BJOG. 2014; 121(9):1097-106.

9. Boonya-ussadorn T, Choi WH, Hyun OJ, Kim SH, Chung SK and Yoo R. 18F-FDG PET/CT Findings in Endometrial Cancer Patients: The Correlation between SUVmax and Clinicopathologic Features. J Med Assoc Thai. 2014;97 (Suppl 2):S115-22. 